\title{
Low Serum 25-Hydroxy Vitamin D (25-OHD) and Hepatic Encephalopathy in HCV-Related Liver Cirrhosis
}

\author{
Mohamed Abd Ellatif Afifi $(\mathbb{D}$, Ahmed Mohamed Hussein, and Mahmoud Rizk
}

Internal Medicine Department, Hepatology and Gastroenterology Unit, Benha University, Egypt

Correspondence should be addressed to Mohamed Abd Ellatif Afifi; dr_malatif82@yahoo.com

Received 10 October 2020; Revised 19 January 2021; Accepted 29 January 2021; Published 13 February 2021

Academic Editor: Dirk Uhlmann

Copyright (C) 2021 Mohamed Abd Ellatif Afifi et al. This is an open access article distributed under the Creative Commons Attribution License, which permits unrestricted use, distribution, and reproduction in any medium, provided the original work is properly cited.

Background. Patients with liver cirrhosis experience a large variety of metabolic disorders associated with more hepatic decompensation. Hepatic encephalopathy $(\mathrm{HE})$ is a significant complication in liver cirrhosis patients, presenting a wide spectrum of neuropsychological symptoms. A deficiency of 25 -hydroxy vitamin D (25-OHD) in the general population is associated with a loss of cognitive function, dementia, and Alzheimer's disease. Aim of the Study. Our study aims to check the relationship between low serum 25-OHD and HE in patients with HCV-related liver cirrhosis and assess its link with patient mortality. Patients and Methods. This study was observationally carried out on 100 patients with HCV-related liver cirrhosis. The patients were divided into 2 groups: Group A-included 50 HCV-related cirrhotic patients with HE, and Group B-included $50 \mathrm{HCV}$-related cirrhotic patients without HE. Assessment of disease severity using the end-stage liver disease (MELD) model and Child Turcotte Pugh (CTP) scores were done, and 25-OHD levels were measured. Comparison of vitamin $\mathrm{D}$ levels in different etiologies and different CTP categories was made using one-way ANOVA. Pearson's correlation between the level of vitamin D and other biomarkers was applied. Results. There was a statistically significant Vitamin D level difference between the two groups. A lower level of vitamin D was observed in the HE group where the severe deficiency was $16 \%$, while it was $6 \%$ in the other group and the moderate deficiency was $24 \%$ in HE group as compared to $10 \%$ in the other group. The insufficient vitamin D level represented $46 \%$ of the non-HE group while none of the HE group falls in this category. Vitamin D level was statistically higher in Grade $1 \mathrm{HE}$ than in Grade 2 which is higher than in Grades 3 to 4 . Vitamin D level was also significantly higher in those who improved from HE as compared to those who died. Conclusion. The lower levels of 25-OHD were associated with the higher incidence of HE in cirrhotic HCV patients. The worsening vitamin D deficiency was associated with increased severity of the liver disease, so vitamin D may be considered a prognostic factor for the severity of liver cirrhosis and high mortality rate in HE patients.

\section{Introduction}

Hepatitis C virus (HCV) infection is a global public health problem. More than 185 million human beings (3\% of the world's population) are infected. Nearly seventy-five percent of HCV patients are living in middle to low-income countries $[1,2]$. Unfortunately, Egypt is one of the countries having the highest global burden of HCV infection with a prevalence of around $4.6 \%$ to $6.8 \%$ [3].

$\mathrm{HCV}$ infection is considered the leading cause of chronic hepatitis, cirrhosis, and hepatocellular carcinoma (HCC). About fifty-five to fifty-eight percent of HCV patients develop fibrosis and cirrhosis and may deteriorate to decompensated liver cirrhosis, HE, and hepatocellular carcinoma [4].

In patients with $\mathrm{HCV}$ cirrhosis, ascitic fluid infection as in spontaneous bacterial peritonitis (SBP) is common and is considered a significant risk factor for circulatory dysfunction and the development of hepatorenal syndrome (HRS) [5-7]. Moreover, disturbance in blood flow to the brain is integrated into the pathogenesis of HE and more likely linked to alterations in the ammonia level and inflammatory status [8].

HE describes a complex variety of neuropsychiatric symptoms ranging from subclinical changes to coma. It is classified according to West Haven criteria into minimal 
(covert) or overt encephalopathy [9]. Up to fifty-five percent of patients with the chronic hepatic disease have been reported with $\mathrm{HE}$ [10]. Symptoms include impaired memory and motor control and decreased levels of energy [11]. HE features can be similar to signs seen in dementia patients [12]. The etiology and pathogenesis of HE are complex and multifactorial, including impaired metabolism of ammonia, dysbiosis that causes inflammation in the intestine and liver [13], decreased levels of circulating branched amino acids [14], abnormalities in electrolyte levels [15], and changes in zinc and manganese levels [16].

Importantly, after treatment, the symptoms and signs of $\mathrm{HE}$ can often be significantly reversed, confirming that it is a functional and not a structural cause of cognitive impairment [17]. HE development presents major challenges for patients and their carers [18]. Until recent times, lactulose has been the major cornerstone of $\mathrm{HE}$ management and is still been used as first-line management to control symptoms of chronic HE and to reverse the symptoms of episodes of acute $\mathrm{HE}$ [19]. The introduction of rifaximin decreased $\mathrm{HE}$ rates and duration of hospital admission and frequency due to $\mathrm{OHE}$ and also improved the quality of life for the patients and their carers [20]. Patients with recurrent episodes of OHE may encounter persistent and cumulative deficits in memory and the ability to learn [21].

Vitamin $\mathrm{D}$ is considered a multifunctional steroid hormone with many actions. Little about Vitamin D's function is actually understood. It is becoming more clear that vitamin $\mathrm{D}$ is not only engaged in calcium homeostasis and bone metabolism but it also has multiple biological actions mediated by vitamin D receptors (VDR) [22] present in even more than thirty tissues [23] including the kidneys, brain, intestine, pituitary, parathyroid gland, prostate, breast, heart muscle, skeletal muscles, hepatic cells, immune system, and endothelial cells [24-28].

Vitamin D is obtained from certain food stuffs and exposure to ultraviolet light. Activation of vitamin D is done through two steps; the first is through hydroxylation of cholecalciferol into the 25-OHD metabolite that occurs in the liver, which is the major circulating metabolic form of vitamin $\mathrm{D}$. It circulates bound to the vitamin $\mathrm{D}$-binding protein (DBP) with a 15-21 day half-life. The second is through an activation process for 1,25 dihydroxy vitamin $\mathrm{D}$ which occurs mainly in the kidney and to a lesser extent in a range of all other tissues such as the bone, mammary gland, brain tissue, monocytes, parathyroid gland, and placenta. This active metabolite has a shorter 10-20-hour half-life. Accordingly, the level of vitamin $\mathrm{D}$ is commonly assessed by measuring circulating 25-OHD [29, 30].

Vitamin $\mathrm{D}$ is an immune modulator and is considered as an anti-infective agent [31]. Clinical associations among vitamin $\mathrm{D}$ status and global and specific areas of cognitive function are increasing, and vitamin D deficiency may also be associated with both depression and schizophrenia [32, 33]. Furthermore, in some areas of cognitive function, vitamin $\mathrm{D}$ deficiency is associated with low mood and impairment of physical performance [34] as well as with a rapid decrease in cognitive function [35].
Most neurons have VDR proteins, including brain glia [36]. Key areas of cognition are the hypothalamus and the brain cortex [37]. The presence of both the VDR protein and vitamin $\mathrm{D}$ metabolites in these brain areas is an indication that the vitamin $\mathrm{D}$ system is involved in the brain's normal function [38].

As the liver is responsible for the first step of hydroxylation of vitamin D into 25-OHD, the levels decrease with more advanced liver disease. Decreased vitamin $\mathrm{D}$ level has been reported in about 92 percent of patients with chronic liver disease (CLD), with a severe 25-OHD deficiency in at least one-third of them $[39,40]$. Also, it is noticed that increased overall mortality in cirrhotic patients is related to a more deficient 25-OHD levels [41].

Our research is aimed at studying the relationship between low serum 25-OHD and HE in HCV-related liver cirrhosis patients and assessing its link to their mortality.

\section{Patients and Methods}

2.1. Study Design. This is a hospital-based noninterventional, prospective, observational study carried out in the internal medicine department (ward-ICU unit) of Benha University Hospitals, Egypt.

2.2. Study Population and Participant Recruitment. The study was conducted on 100 patients with HCV-related liver cirrhosis who were divided into 2 groups: Group A-including $50 \mathrm{HCV}$-related cirrhotic patients with $\mathrm{HE}$, and Group $\mathrm{B}$-including $50 \mathrm{HCV}$-related cirrhotic patients without $\mathrm{HE}$ or other liver cirrhosis-related complications (Figure 1).

2.2.1. Inclusion Criteria. The following are the inclusion criteria:

(i) Patients of both sexes aged 18 years or more

(ii) Eligible patients who had a diagnosis of liver cirrhosis secondary to $\mathrm{HCV}$ infection

(iii) Diagnosis of liver cirrhosis based upon clinical, laboratory, and U/S features

2.2.2. Exclusion Criteria. The following are the inclusion criteria:

(i) Age $<18$ years

(ii) Patients with liver cirrhosis secondary to causes other than HCV infection

(iii) Patients with a history of vitamin D supplementation in the previous 2 months

(iv) Patients with a history of a wide variety of medications including that lead to low vitamin D levels (e.g., antifungal, anticonvulsant, or glucocorticoid medications).

(v) Patients with vitamin D deficiency due to other causes like (limited sunlight exposure, CKD, IBD, 


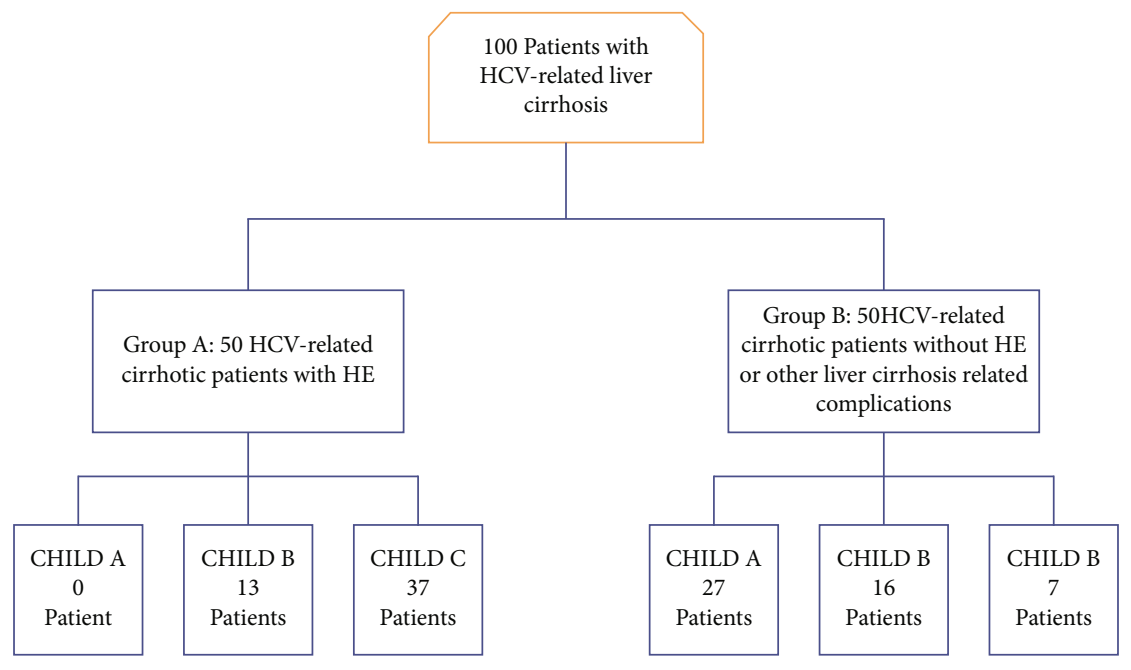

Figure 1: Patient selection flowchart (Afifi, Hussein, and Rizk, 2020).

malabsorption syndrome, obstructive jaundice, lymphomas, or hyperparathyroidism)

(vi) Patients with HCC

2.3. Consent. Written informed consent was obtained from all individual participants included in the study. For patients with altered consciousness, informed consent was obtained from their first-degree relatives.

2.4. Patients Were Subjected to. The patients were subjected to the following:

(i) A detailed history taking and a clinical examination for stigmata of CLD including ascites and presence of $\mathrm{HE}$; assessment of the severity of patients presenting with HE done according to West Haven criteria [42]

(ii) An assessment of disease severity using a model for end-stage liver disease (MELD) [43] and CTP scores [44]

(iii) Biochemical parameters $(\mathrm{CBC}$, albumin, bilirubin, INR, serum creatinine, serum sodium, serum potassium, and alpha fetoprotein L3)

(iv) Pelvi-abdominal U/S to detect radiological features of cirrhosis (irregular surface, coarse texture, and attenuated hepatic veins) and ascites and to exclude hepatic focal lesions

(v) A diagnostic abdominal paracentesis of patients with ascites to obtain an ascetic fluid sample for polymorphonuclear neutrophil (PMN) count and ascitic fluid culture for diagnosis of SBP

A combination of the BacT/Alert Microbial Detection System with the VITEK 2 system (bioMérieux, France) was used to achieve rapid bacterial identification and susceptibility testing in the diagnosis of SBP $[45,46]$.
2.5. Measuring 25-OH Levels. 25-OH levels were measured using the Diasorin RIA $^{\circledR}$ assay technique. $25-\mathrm{OH}$ vitamin D status was defined as sufficient $(>75 \mathrm{nmol} / \mathrm{L})$, insufficient (50-75 nmol/L), mild deficiency $(25-50 \mathrm{nmol} / \mathrm{L})$, moderate deficiency $(12.5-25 \mathrm{nmol} / \mathrm{L})$, and severe deficiency $(<12.5 \mathrm{nmol} / \mathrm{L})[47,48]$.

2.6. Statistical Methods and Data Analysis. Descriptive statistics for the two groups were presented as the mean and standard deviation for numerical variables and as frequencies and cumulative frequencies for categorical variables. A comparison of differences between the two groups was done using the independent $t$-test for numerical variables, and using the chi-square test as well as Fisher's exact test for categorical variables. The comparison of vitamin $\mathrm{D}$ levels in different $\mathrm{HE}$ etiologies and different CTP categories was made using one-way ANOVA. The comparison of 25OHD levels in different SBP categories and different HE grades was made using the Kruskal-Wallis Test. The correlation between the level of vitamin $\mathrm{D}$ and other biomarkers was made using Pearson's correlation.

\section{Results}

3.1. Patient Characteristics and Biochemical Analysis. Among the enrolled 100 patients, Group A included 50 patients having HCV-related cirrhosis complicated with HE (13 patients Child B and 37 patients Child C) and Group B included 50 patients having HCV-related cirrhosis without HE (27 patients Child A, 16 patients Child B, and 7 patients Child C) (Figure 1). Baseline characteristics were compared in both groups showing nonsignificant difference regarding age. $\mathrm{BMI}$ was higher for the $\mathrm{HE}$ group (mean $=28.7, \mathrm{SD}=$ 3.5 ) than the non-HE group ( mean $=25.7, \mathrm{SD}=3.1)$. The difference of potassium level and WBCs count in both groups was not significant. There was a significant difference between both groups in other variables. MELD score and CTP score were higher in the HE group $(21.8 \pm 5.6)$ and $(11.8 \pm 2.4)$, respectively, versus $(12.6 \pm 5.9)$ for MELD and $(7.0 \pm 2.1)$ for CTP in the non-HE group. Moreover, 
TABLE 1: Characteristics and biomarkers in both groups.

\begin{tabular}{|c|c|c|c|c|c|}
\hline & \multicolumn{2}{|c|}{ Group $1(\mathrm{HE})$} & \multicolumn{2}{|c|}{ Group 2 (non-HE) } & \multirow{2}{*}{$P$ value } \\
\hline & Mean & SD & Mean & SD & \\
\hline Age (years) & 51.1 & 6.2 & 50.6 & 7.2 & 0.733 \\
\hline BMI $\left(\mathrm{kg} / \mathrm{m}^{2}\right)$ & 28.7 & 3.5 & 25.7 & 3.1 & $<0.001$ \\
\hline 25-OHD (nmol/L) & 27.2 & 11.9 & 41.0 & 15.2 & $<0.001$ \\
\hline Bilirubin (mg/dL) & 3.7 & 1.3 & 2.2 & 0.9 & $<0.001$ \\
\hline Creatinine $(\mathrm{mg} / \mathrm{dL})$ & 1.1 & 0.5 & 0.8 & 0.4 & 0.001 \\
\hline Albumin (mg/dL) & 2.9 & 0.3 & 3.5 & 0.4 & $<0.001$ \\
\hline INR & 1.9 & 0.3 & 1.2 & 0.3 & $<0.001$ \\
\hline Sodium (mEq/L) & 133.9 & 3.8 & 137.4 & 3.8 & $<0.001$ \\
\hline Potassium $(\mathrm{mEq} / \mathrm{L})$ & 4.2 & 0.8 & 4.1 & 0.6 & 0.521 \\
\hline $\mathrm{HB}(\mathrm{g} / \mathrm{dL})$ & 10.3 & 1.5 & 11.9 & 2.0 & $<0.001$ \\
\hline $\mathrm{WBC} \times 10^{6}$ & 5.8 & 2.9 & 6.5 & 2.4 & 0.158 \\
\hline $\mathrm{PLT} \times 10^{3}$ & 111.3 & 30.4 & 151.3 & 28.5 & $<0.001$ \\
\hline CTP score & 11.8 & 2.4 & 7.0 & 2.1 & $<0.001$ \\
\hline MELD score & 21.8 & 5.6 & 12.6 & 5.9 & $<0.001$ \\
\hline
\end{tabular}

Summary of the results of the descriptive study of demographic, laboratory, and clinical variables included in the CTP and MELD scores. BMI: body mass index; 25-OHD: 25-hydroxy vitamin D; INR: international normalized ratio; HB: hemoglobin; WBC: white blood cell count; PLT: platelet count; CTP: Child Turcotte Pugh; MELD: model for end Stage liver disease. The independent $t$-test was used.

bilirubin, creatinine, and INR were higher in the HE group, while albumin, sodium, hemoglobin, and platelets were lower in the HE group (Table 1).

\subsection{Comparing Both Groups as regards Other Categorical Variables (Table 2)}

3.2.1. Sex. There was no significant difference between both groups regarding sex (group 1 included 38 males and 12 females, while group 2 included 33 males and 17 females with $P$ value $=0.271)$.

3.2.2. CTP Classification. None of the HE group was classified as $\mathrm{A}$, while $54 \%$ of the non-HE group were classified as A, and $17 \%$ of this group were classified as $C$ while $74 \%$ of the $\mathrm{HE}$ group were classified as $\mathrm{C}$, and this difference was statistically significant.

3.2.3. Ascites. $6 \%$ of the HE group had no ascites as compared to $54 \%$ of the non-HE group while $54 \%$ of the HE group had poorly controlled ascites as compared to $20 \%$ of the non-HE group and this difference was statistically significant.

3.2.4. Other Variables. Comparing both groups regarding SBP, HRS, and hematemesis and/or melena $(\mathrm{H} / \mathrm{M})$ was statistically significant. The entire non-HE group did not have SBP, HRS, or H/M. Thirty percent of the HE group prognosis was death as compared to $0 \%$ in the non-HE group.

3.3. Vitamin 25-OHD Levels in Both Groups. A higher degree of 25-OHD deficiency was observed in the HE group where the severe deficiency was $16 \%$, as compared to $6 \%$ in the other group, and the moderate deficiency was $24 \%$ as compared to $10 \%$ in the other group. The insufficient $25-\mathrm{OHD}$ level represented $46 \%$ of the non-HE group while none of the HE group falls in this category. Differences in 25-OHD levels between both groups were highly significant (Table 3 ).

3.4. Etiology of HE. The most common etiology of HE was the SBP (32\%), followed by H/M (20\%), HRS (18\%), and hypokalemia (14\%), while others' etiologies represented 16\% (Table 4).

3.5. Comparing 25-OHD Levels in Different HE Etiologies. The levels of 25-OHD in the SBP and HRS groups were less than those in the H/M group which were less than those in the hypokalemia as shown in (Table 5, Figure 2).

3.6. Comparing 25-OHD Levels in Different SBP Categories, Child Classes, and the Correlation with MELD Score. The levels of 25-OHD in the SBP (classic and monobacterial (MNB) groups were less than the culture-negative neutrocytic ascites (CNNA)) group, and this difference was statistically significant (Table 6 and Figure 3). The level of 25OHD in Child A was higher than Child B and Child B was higher than Child $\mathrm{C}$ (Table 7, Figure 4). There was a strong negative correlation $(r=-0.91)$ between the 25-OHD level and the MELD score (Table 8, Figure 5).

3.7. Comparing 25-OHD Levels in Different HE Grades. The level of 25-OHD in Grade 1 was higher than that in Grade 2 which was higher than that in Grades 3 to 4 , and this difference was statistically significant (Table 9, Figure 6).

3.8. Comparing 25-OHD Levels in relation to Prognosis. Comparing 25-OHD levels between those who died (mean 15.1 \pm 7.5 ) and those improved in the HE group (mean $32.5 \pm$ 9.5) revealed that 25-OHD levels were significantly higher for those who improved as compared to those who died (Table 10). ROC curve for 25-OHD levels showed the cutoff value $18.5(\mathrm{nmol} / \mathrm{L})$ to predict improvement in the $\mathrm{HE}$ 
TABLE 2: Other characteristics in both groups.

\begin{tabular}{|c|c|c|c|c|c|}
\hline & & & & & \\
\hline & & & $1(\mathrm{HE})$ & 2 (non-HE) & $P$ value \\
\hline & Male & $N$ & 38 & 33 & \\
\hline Sex & Hidic & $\%$ & $76.0 \%$ & $66.0 \%$ & \\
\hline $\operatorname{sex}$ & Female & $N$ & 12 & 17 & 0.211 \\
\hline & Tetinare & $\%$ & $24.0 \%$ & $34.0 \%$ & \\
\hline & A & $N$ & 0 & 27 & \\
\hline & 1 & $\%$ & $0.0 \%$ & $54.0 \%$ & \\
\hline CTP & $P_{2}$ & $N$ & 13 & 16 & -0001 \\
\hline C11 & $D$ & $\%$ & $26.0 \%$ & $32.0 \%$ & 20.001 \\
\hline & $C$ & $N$ & 37 & 7 & \\
\hline & C & $\%$ & $74.0 \%$ & $14.0 \%$ & \\
\hline & None & $N$ & 3 & 27 & \\
\hline & 19010 & $\%$ & $6.0 \%$ & $54.0 \%$ & \\
\hline Ascites & Medically controlled & $N$ & 20 & 13 & $<0001$ \\
\hline & 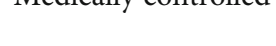 & $\%$ & $40.0 \%$ & $26.0 \%$ & -0.001 \\
\hline & Poorly controlled & $N$ & 27 & 10 & \\
\hline & 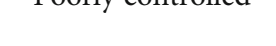 & $\%$ & $54.0 \%$ & $20.0 \%$ & \\
\hline & $\mathrm{No}$ & $N$ & 34 & 50 & \\
\hline & $1 \mathrm{NO}$ & $\%$ & $68.0 \%$ & $100.0 \%$ & \\
\hline & SRP (closcis) & $N$ & 5 & 0 & \\
\hline SBP & (Chasole) & $\%$ & $10.0 \%$ & $0.0 \%$ & $<0.001$ \\
\hline & SBP (CNNA) & $N$ & 8 & 0 & \\
\hline & 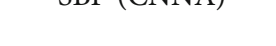 & $\%$ & $16.0 \%$ & $0.0 \%$ & \\
\hline & SBP (MNB) & $N$ & 3 & 0 & \\
\hline & GDi (IVIVD) & $\%$ & $6.0 \%$ & $0.0 \%$ & \\
\hline & HRS & $N$ & 9 & 0 & \\
\hline HRS & & $\%$ & $18.00 \%$ & $0.00 \%$ & 0.003 \\
\hline & $\mathrm{No}$ & $N$ & 41 & 50 & \\
\hline & $1 \mathrm{NO}$ & $\%$ & $82.00 \%$ & $100.00 \%$ & \\
\hline & No & $N$ & 40 & 50 & \\
\hline $\mathrm{H} / \mathrm{M}$ & 10 & $\%$ & $80.0 \%$ & $100.0 \%$ & 0.001 \\
\hline $11 / 1 \mathrm{N1}$ & Yes & $N$ & 10 & 0 & \\
\hline & 100 & $\%$ & $20.0 \%$ & $0.0 \%$ & \\
\hline & Death & $N$ & 15 & 0 & \\
\hline Proonosis & Dentist & $\%$ & $30.00 \%$ & $0.00 \%$ & $<0001$ \\
\hline 1 10gindis & Imnroved & $N$ & 35 & 0 & -0.001 \\
\hline & 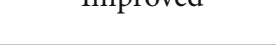 & $\%$ & $70.00 \%$ & $0.00 \%$ & \\
\hline
\end{tabular}

Summary of the results of the descriptive study of (demographic, laboratory, and clinical variables) included in the CTP and MELD scores. CTP: Child Turcotte Pugh; SBP: spontaneous bacterial peritonitis; HRS: heptorenal syndrome; H/M: hematemesis and/or melena. The chi-square test and Fisher's exact tests were used.

group (patients who recovered and did not die) with a sensitivity of $94 \%$ and a specificity of $80 \%$ (Figure 7 ). By using $18.5(\mathrm{nmol} / \mathrm{L})$ as a cut-off level for 25-OHD for survival in the HE group, it showed a sensitivity of $94 \%$ and a specificity of $80 \%$ (Table 11 ).

3.9. Correlation between 25-OHD Level in the HE Group and Other Variables. There was a strong negative correlation between bilirubin $(r=-0.85, \quad P=<0.001)$, creatinine $(r=-0.55, P=<0.001)$, INR $(R=-0.65, P=<0.001)$, and potassium $(r=-0.39, P=<0.005)$ levels and serum 25-OHD level. Strong positive correlation was found between albumin $(r=0.81, P=<0.001)$ and sodium $(r=0.71, P=<0.001)$ levels and serum 25-OHD level. No correlation was found between 25-OHD level and hemoglobin level, WBCs, or platelet count (Table 12). 
TABLE 3: Comparison of 25-OHD levels between the two groups.

\begin{tabular}{|c|c|c|c|c|}
\hline \multirow{2}{*}{ 25-OHD level } & & \multicolumn{2}{|c|}{ Groups } & \multirow{2}{*}{$P$ value } \\
\hline & & 1 (HE) & 2 (non-HE) & \\
\hline \multirow{2}{*}{ Insufficient } & $N$ & 0 & 23 & \multirow{8}{*}{$<0.001$} \\
\hline & $\%$ & $0.00 \%$ & $46.00 \%$ & \\
\hline \multirow{2}{*}{ Mild deficiency } & $N$ & 30 & 19 & \\
\hline & $\%$ & $60.00 \%$ & $38.00 \%$ & \\
\hline \multirow{2}{*}{ Moderate deficiency } & $N$ & 12 & 5 & \\
\hline & $\%$ & $24.00 \%$ & $10.00 \%$ & \\
\hline \multirow{2}{*}{ Severe deficiency } & $N$ & 8 & 3 & \\
\hline & $\%$ & $16.00 \%$ & $6.00 \%$ & \\
\hline
\end{tabular}

The more 25-OHD deficiency was noticed in the HE group. The chi-square test was used.

TABle 4: Common etiologies of HE in Group 1.

\begin{tabular}{lcc}
\hline Etiology of HE & $N$ & $\%$ \\
\hline SBP & 16 & $32.0 \%$ \\
HRS & 9 & $18.0 \%$ \\
H/M & 10 & $20.0 \%$ \\
Hypokalemia & 7 & $14.0 \%$ \\
Other & 8 & $16.0 \%$ \\
Total & 50 & 100.0 \\
\hline
\end{tabular}

SBP was the most common etiology in the HE group. SBP: spontaneous bacterial peritonitis; HRS: heptorenal syndrome; H/M: hematemesis and/or melena; Other: means other causes of HE-like infections (other than SBP), constipation, diarrhea, or even undetermined causes.

3.10. Studying Multiple Logistic Regression for Serum 25$\mathrm{OHD}$ and Serum Albumin Levels. There was a positive OR for 25-OHD, supporting our theory that the low serum level of 25-OHD is associated with increased severity of the liver disease and HE (Table 13).

\section{Discussion}

Vitamin D deficiency is frequently found in cirrhotic patients. The liver is an essential organ for the biosynthesis of vitamin $\mathrm{D}$, where it is the main organ for the production of 25-OHD [39]. The reduced number of liver cells, decreased exposure to sunlight, greatly reduced adipose tissue mass, impaired vitamin D absorption, and hydroxylation in the liver are all reasons for the low vitamin D levels in cirrhotic patients [49].

We tried in our research to study the relationship between low serum 25-OHD and $\mathrm{HE}$ in patients with $\mathrm{HCV}$-related liver cirrhosis and to evaluate whether there is a link between low serum 25OHD and mortality rate.

The study revealed a statistically significant correlation between the severity of cirrhosis (CTP and MELD scores) and low serum levels of 25-OHD. We found that 25OHD levels were negatively correlated with both MELD and CTP scores, suggesting that as the disease advances, 25OHD levels become more deficient $(P<0.001)$. CTP class $C$ contained more $25 \mathrm{OHD}$-deficient cases than class $\mathrm{B}$ or class $\mathrm{A}$ as the level of vitamin $\mathrm{D}$ in the Child A group $(53.3 \pm 4.4 \mathrm{nmol} / \mathrm{L})$ was higher than in the Child B group $(35.9 \pm 7.2 \mathrm{nmol} / \mathrm{L})$ while that in the Child B group is higher than in Child $C$ $(21.2 \pm 9.1 \mathrm{nmol} / \mathrm{L})$ group. Similar results were seen in a study by Fisher et al., in which more vitamin D-deficient cases were found in class $\mathrm{C}$ [50].

Moreover, the results of our study were similar to many other studies which have shown that vitamin $\mathrm{D}$ level is inversely correlated to the CTP score and MELD [51]. Two studies were conducted by Fernández et al. and Zhao et al. and found that vitamin D decreases further as cirrhosis of the liver becomes more advanced. Thus, patients with higher CTP and MELD scores had notably lower vitamin D levels compared to patients with lower CTP and MELD scores [52, 53].

The study also showed that the serum 25-OHD level was significantly different between the three SBP groups $(P<0.001)$. The serum 25-OHD level was significantly lower in patients with classic SBP $(13 \pm 3.4 \mathrm{nmol} / \mathrm{L})$ and $\mathrm{MNB}$ $(13.5 \pm 4.9 \mathrm{nmol} / \mathrm{L})$ compared to patients with CNNA $(23.5 \pm 3 \mathrm{nmol} / \mathrm{L}, P<0.001)$.

Vitamin D induces synthesis of LL-37 in human macrophages, which is really the only antibacterial peptide controlled by vitamin D in humans [39]. Notably, insufficient production of LL-37 in the peritoneal cavity induced by vitamin $\mathrm{D}$ deficiency in patients with cirrhosis and ascites has been hypothesized to contribute to an increased risk of SBP with increments in the mortality rates [54].

Our study showed that there was a statistically significant difference in vitamin $\mathrm{D}$ levels between both groups $(P<0.001)$. As regards the comparison of vitamin $\mathrm{D}$ levels in different $\mathrm{HE}$ groups, there was a statistically significant difference $(P<0.001)$ as the level of vitamin D in Grade 1 is higher than that in Grade 2 that is higher than that in Grades 3 to 4 . This is in agreement with two studies done by Vidot et al. and Iruzubieta who stated that the lower the level of 25-OHD, the higher the HE grade. Deficient antiinflammatory properties of 25-OHD can explain this association. Systemic inflammation and changes in hepatic metabolism such as elevated levels of ammonia are considered HE precipitants and exacerbate the current $\mathrm{HE}[55,56]$.

In a study done by Stokes et al., it was unclear whether there is a steady decline in brain function as the 25-OHD 
TABLE 5: Comparison of 25-OHD level in different HE etiologies.

\begin{tabular}{|c|c|c|c|c|c|}
\hline & $N$ & Mean & SD & $P$ value & Comparisons \\
\hline SBP & 16 & 18.4 & 6.1 & & \\
\hline HRS & 9 & 16.8 & 8.2 & & \\
\hline $\mathrm{H} \& / \mathrm{M}$ & 10 & 29.3 & 3.7 & $<0.001$ & SBP \& HRS $<(H / M)<$ hypokalemia \\
\hline Hypokalemia & 7 & 44.9 & 2.5 & & \\
\hline Other & 8 & 41.6 & 5.3 & & \\
\hline
\end{tabular}

Comparison of 25-OHD levels in patients with HE due to different etiologies. Patients with HE due to SBP and HRS showed the least serum 25-OHD levels followed by 25-OHD levels in HE patients due to H/M and hypokalemia. SBP: spontaneous bacterial peritonitis; HRS: heptorenal syndrome; H\&/M: mematemesis and/or melena; Other: means other causes of HE-like infections (other than SBP), constipation, diarrhea, or even undetermined causes. The one-way ANOVA test was used.

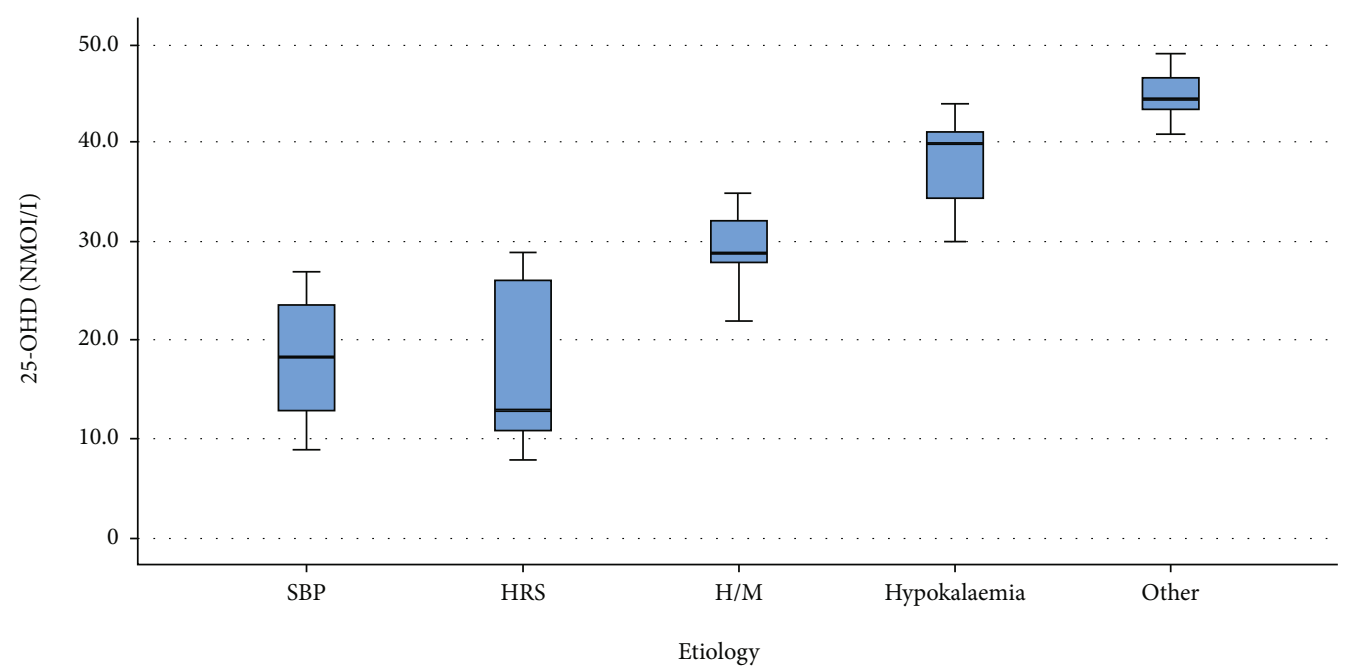

Figure 2: Comparison of 25-OHD levels in different HE etiologies (Afifi, Hussein, and Rizk, 2020).

TABLE 6: Comparison of 25-OHD level in different SBP categories.

\begin{tabular}{lccccc}
\hline SBP & $N$ & Mean & SD & $P$ value & Comparisons \\
\hline SBP(classic) & 5 & 13.0 & 3.4 & & SBP (classic and MNB) $<$ SBP $($ CNNA) \\
SBP(CNNA) & 8 & 23.5 & 3.0 & $<0.001$ & \\
SBP(MNB) & 2 & 13.5 & 4.9 & & \\
\hline
\end{tabular}

The levels of 25-OHD in different SBP categories were illustrated. The lowest levels were detected in classic type, followed by MNB and CNNA types. SBP: spontaneous bacterial peritonitis; CNNA; culture-negative neutrocytic ascites; MNB: monobacterial. The Kruskal-Wallis test was used.

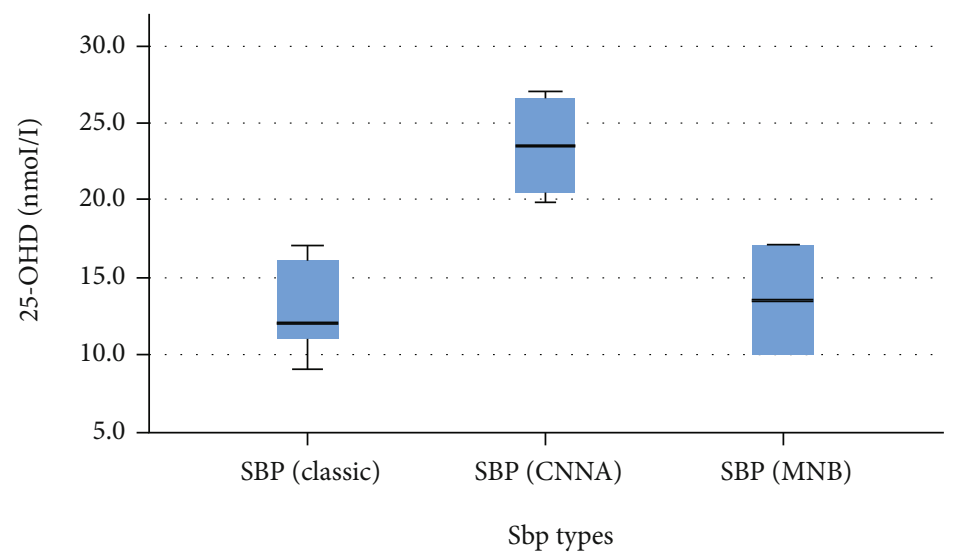

Figure 3: Comparison of 25-OHD levels in different SBP categories (Afifi, Hussein, and Rizk, 2020). 
TABLE 7: Comparison of 25-OHD levels in different CTP categories.

\begin{tabular}{llclll}
\hline CTP & N & Mean & SD & P-value & Comparisons \\
\hline A & 27 & 53.3 & 4.4 & & $<0.001$ \\
B & 29 & 35.9 & 7.2 & & A $>$ B $>$ C \\
C & 44 & 21.2 & 9.1 & & \\
\hline
\end{tabular}

Comparison of 25-OHD levels in different CTP categories. Levels were decreased from CTP Grade A to Grades B and C. CTP: Child Turcotte Pugh. The oneway ANOVA test was used.

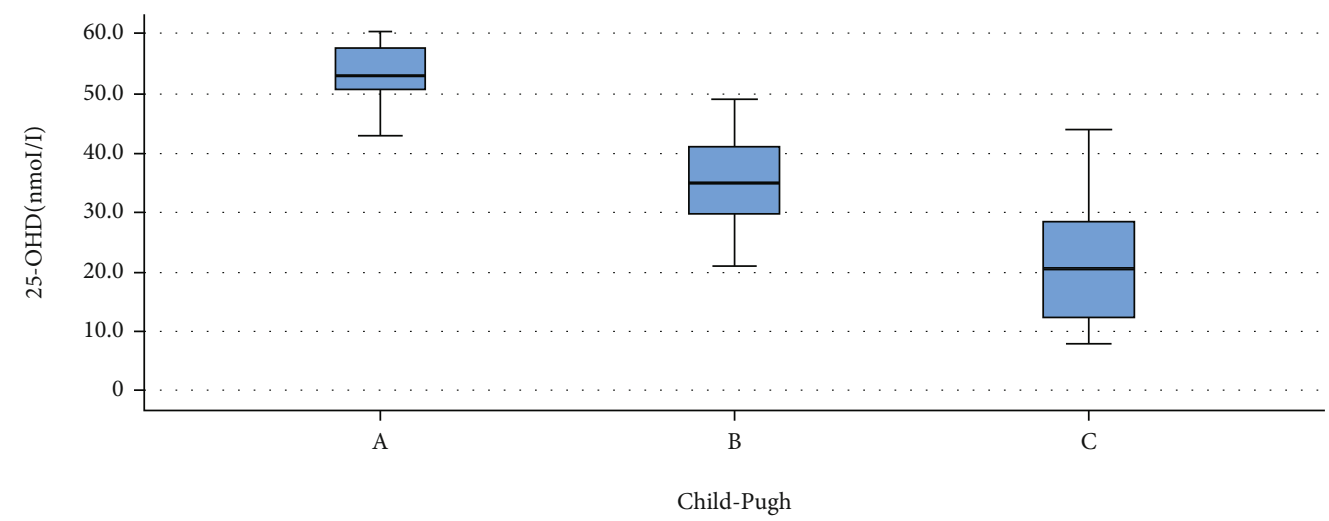

FIgURe 4: Comparison of 25-OHD in different CTP categories (Afifi, Hussein, and Rizk, 2020).

TABLE 8: Correlation between 25-OHD level and MELD score.

\begin{tabular}{lcc}
\hline & & MELD score \\
\hline & Pearson correlation & -0.909 \\
$25-O H D(\mathrm{nmol} / \mathrm{L})$ & $P$ value & $<0.001$ \\
& $N$ & 100 \\
\hline
\end{tabular}

A highly significant negative correlation between 25-OHD levels and MELD scores was detected. Pearson's correlation test was used.

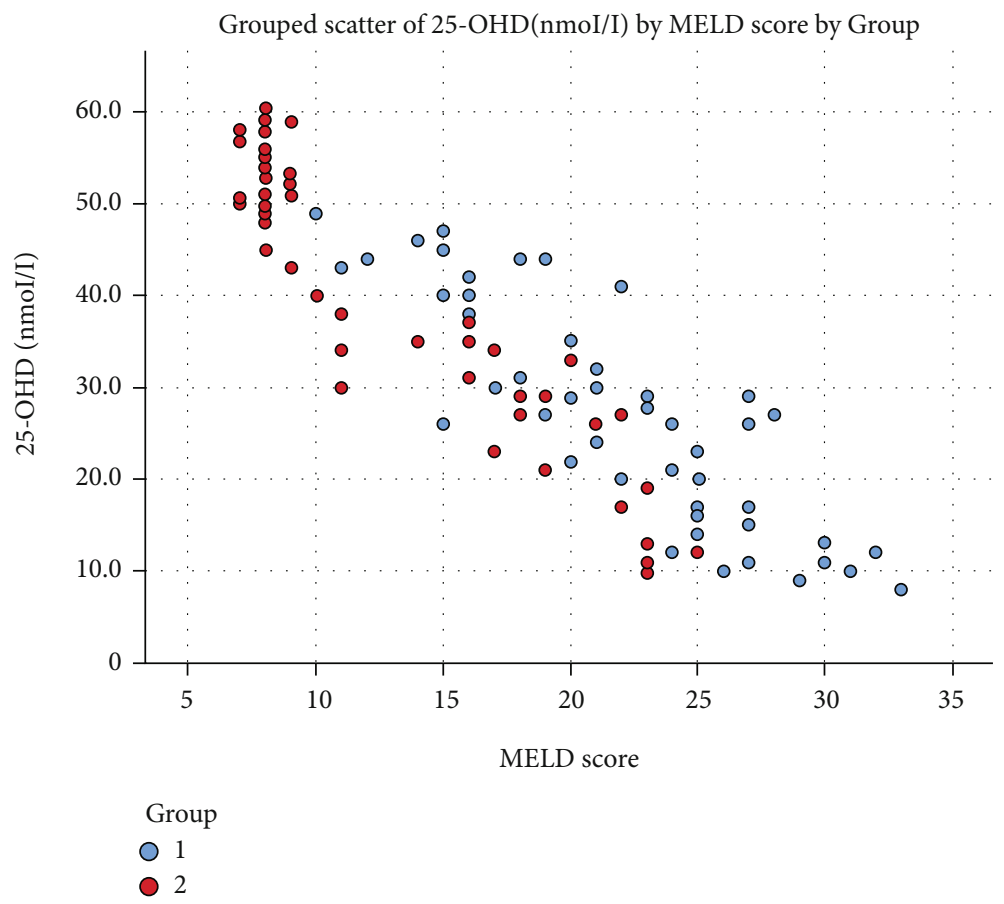

FIGURE 5: Grouped scatter plot showing the correlation between the 25-OHD level and MELD score (Afifi, Hussein, and Rizk, 2020). 
TABLE 9: Comparison of 25-OHD level in different HE grades.

\begin{tabular}{llllll}
\hline HE grade & $N$ & Mean & SD & $P$ value & Comparisons \\
\hline Grade 1 & 12 & 43.8 & 2.8 & & Grade 1 $>$ Grade 2 $>$ Grades 3 to 4 \\
Grade 2 & 18 & 29.6 & 3.3 & 5.0 & \\
Grade 3 to 4 & 20 & 15.3 & & \\
\hline
\end{tabular}

The 25-OHD level is significantly lower in higher grades of HE. The Kruskal-Wallis test was used.

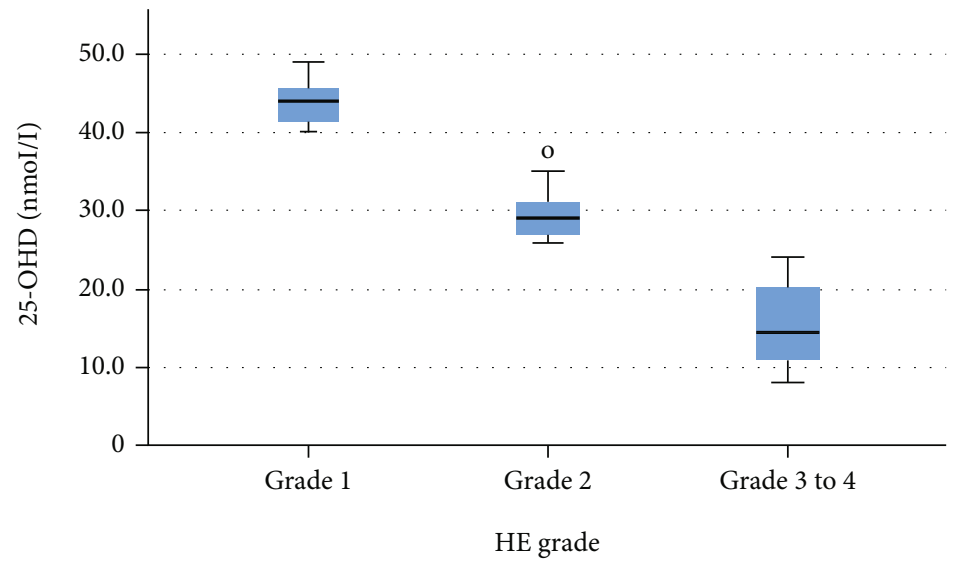

Figure 6: Comparison of 25-OHD level in different HE grades (Afifi, Hussein, and Rizk, 2020).

TABLE 10: Comparison of 25-OHD levels in those who died and those improved in the HE group.

\begin{tabular}{lcccc}
\hline Prognosis & $N$ & Mean & SD & $P$ value \\
\hline Died & 15 & 15.1 & 7.1 & $<0.001$ \\
Improved & 35 & 32.5 & 9.6 & \\
\hline
\end{tabular}

The level of 25-OHD was significantly lower in HE patients who died. The independent $t$-test was used.

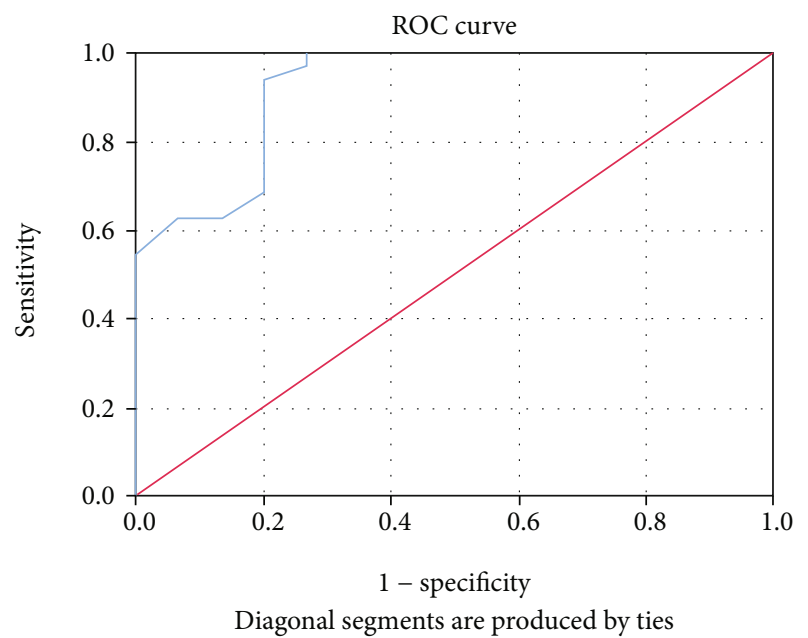

FIGURE 7: ROC curve for the 25-OHD level to predict improvement in HE group (patients who have recovered and did not die) (Afifi, Hussein, and Rizk, 2020).
TABLE 11: Showing cut-off values of 25-OHD for survival in the HE group.

\begin{tabular}{|c|c|c|}
\hline Coordinates of the curve & & \\
\hline Test result variable(s): $25-\mathrm{OHD}(\mathrm{nn}$ & $\mathrm{l} / \mathrm{L})$ & \\
\hline Positive if greater than or equal to & Sensitivity & $1-$ specificity \\
\hline 7.000 & 1.000 & 1.000 \\
\hline 8.500 & 1.000 & 0.933 \\
\hline 9.500 & 1.000 & 0.867 \\
\hline 10.500 & 1.000 & 0.733 \\
\hline 11.500 & 1.000 & 0.600 \\
\hline 12.500 & 1.000 & 0.467 \\
\hline 13.500 & 1.000 & 0.400 \\
\hline 14.500 & 1.000 & 0.333 \\
\hline 15.500 & 1.000 & 0.267 \\
\hline 16.500 & 0.971 & 0.267 \\
\hline 18.500 & 0.943 & 0.200 \\
\hline 20.500 & 0.886 & 0.200 \\
\hline 21.500 & 0.857 & 0.200 \\
\hline 22.500 & 0.829 & 0.200 \\
\hline 23.500 & 0.800 & 0.200 \\
\hline 25.000 & 0.771 & 0.200 \\
\hline 26.500 & 0.686 & 0.200 \\
\hline 27.500 & 0.629 & 0.133 \\
\hline 28.500 & 0.629 & 0.067 \\
\hline 29.500 & 0.543 & 0.000 \\
\hline 30.500 & 0.486 & 0.000 \\
\hline 31.500 & 0.457 & 0.000 \\
\hline
\end{tabular}

Cutoff values of 25-OHD for survival in the HE group: cutoff value 18.5 (nmol/L) of 25-OHD level was detected to predict improvement in the HE group with a sensitivity of $94 \%$ and a specificity of $80 \%$. 
TABLE 12: Correlation between 25-OHD levels and other biochemical parameters in the HE group.

\begin{tabular}{lccccccccc}
\hline & Bilirubin & Creatinine & Albumin & INR & Sodium & Potassium & HB & WBC & PLT \\
\hline Pearson correlation & -0.846 & -0.55 & 0.811 & -0.653 & 0.708 & -0.39 & 0.203 & -0.166 & 0.276 \\
P-value & $<0.001$ & $<0.001$ & $<0.001$ & $<0.001$ & $<0.001$ & 0.005 & 0.158 & 0.249 & 0.052 \\
$\mathrm{~N}$ & 50 & 50 & 50 & 50 & 50 & 50 & 50 & 50 & 50 \\
\hline
\end{tabular}

The table is showing a strong negative correlation in the HE group, between bilirubin level, creatinine, INR, and potassium and serum 25 -OHD. A strong positive correlation was detected between albumin level and sodium level and serum 25-OHD level. No correlation was found between 25-OHD level and hemoglobin level, WBCs, or platelet count. Pearson's correlation test was used.

TABLE 13: Multiple logistic regression for the serum 25-OHD and serum Albumin levels in association with HE.

\begin{tabular}{lcccc}
\hline & \multirow{2}{*}{ OR } & \multirow{2}{*}{$P$ value } & \multicolumn{2}{c}{$95 \%$ CI for OR } \\
& & & Lower & Upper \\
\hline 25-OHD (nmol/L) & 1.063 & $<0.002$ & 1.027 & 1.109 \\
Albumin & 12591.328 & $<0.001$ & 178.757 & 886908.689
\end{tabular}

The table is showing multiple logistic regression for the serum $25-\mathrm{OHD}$ levels and serum albumin levels with positive OR, confirming our theory that low serum level of 25-OHD is associated with increased severity of the liver disease and HE.

levels drop or whether there is a threshold at which point there is a significant reduction in brain function in patients with cirrhosis. To show a causative relationship of vitamin $\mathrm{D}$ with $\mathrm{HE}$, it was necessary to examine the effects of vitamin D supplementation on encephalopathy symptoms in patients with CLD. The literature suggests that it is an association, not causality. There was a sufficient association between cognitive and behavioral changes associated with 25-OHD deficiency to suggest a role for vitamin $\mathrm{D}$ deficiency in the development of HE in patients with liver cirrhosis [41].

In our study, a high mortality rate was associated with serum 25-OHD deficiency. A comparison of vitamin D levels in those who died and those who were improved in the HE group showed that there was a statistically significant difference. Vitamin D level was higher in patients who were improved (35 patients) with a mean 25-OHD level $(32.5 \mathrm{nmol} / \mathrm{L})$ in comparison with those who died (15 patients) with a mean $25-\mathrm{OHD}$ level $(15.1 \mathrm{nmol} / \mathrm{L})$ and $(P<0.001)$. Because patients with cirrhosis had lower serum 25-OHD levels, we suggested that 25-OHD levels might be of prognostic value in these patients $[41,57,58]$. The ROC analysis showed that the cut-off level of vitamin $\mathrm{D}>18.5 \mathrm{nmol} / \mathrm{L}$ can predict the survival in $\mathrm{HE}$ with a sensitivity of $94 \%$ and a specificity of $80 \%$ and this is in concordance with a study conducted by Paternostro et al. which reported a high mortality rate in cirrhotic patients with serum 25-OH D levels less than $6 \mathrm{ng} / \mathrm{mL}$ (HR 1.723, $P=0.013$ ) [59].

Recently, several studies have found the association between serum albumin level and $\mathrm{HE}$ and role of human albumin infusion for prevention and treatment of HE [60]. Our study showed that multiple logistic regression for the serum 25-OHD and serum albumin levels in association with $\mathrm{HE}$ revealed positive $\mathrm{OR}$, confirming our theory that the low serum level of 25-OHD is associated with increased severity of the liver disease and HE.
Finally, we hope that our study will help physicians understand that maintaining normal vitamin $\mathrm{D}$ levels will provide a better prognosis and a better quality of life for CLD patients.

\section{Limitations}

The main limitation of the study is the sample size. A larger study population is essential for the best results.

\section{Conclusion}

Vitamin D deficiency was common in the majority of patients with CLD. Low levels of 25-OHD were associated with HE in patients with HCV-related liver cirrhosis. The worsening vitamin $\mathrm{D}$ deficiency was associated with increased severity of liver disease so it may be considered a prognostic parameter for the severity of liver cirrhosis. In addition, it may be linked to a higher mortality rate in the HE group.

\section{Data Availability}

The datasets used and analyzed during the current study are available from the corresponding author on reasonable request.

\section{Ethical Approval}

The study obtained approval.

\section{Consent}

All participants have provided written consent.

\section{Conflicts of Interest}

The authors declare that they have no conflicts of interest.

\section{References}

[1] A. Petruzziello, S. Marigliano, G. Loquercio, A. Cozzolino, and C. Cacciapuoti, "Global epidemiology of hepatitis C virus infection: an up-date of the distribution and circulation of hepatitis C virus genotypes," World Journal of Gastroenterology, vol. 22, no. 34, pp. 7824-7840, 2016.

[2] K. Mohd Hanafiah, J. Groeger, A. D. Flaxman, and S. T. Wiersma, "Global epidemiology of hepatitis $\mathrm{C}$ virus infection: new estimates of age-specific antibody to HCV seroprevalence," Hepatology, vol. 57, no. 4, pp. 1333-1342, 2013. 
[3] World Health Organization, Key Facts on Hepatitis C Treatment, World Health Organization, Geneva, 2016.

[4] WHO, Hepatitis C fact sheet no. 164 [updated July 2015], WHO Special Programme for Research and Training in Tropical Diseases (TDR), 2016.

[5] S. Møller, F. Bendtsen, and J. H. Henriksen, "Pathophysiological basis of pharmacotherapy in the hepatorenal syndrome," Scandinavian Journal of Gastroenterology, vol. 40, pp. 491500, 2005.

[6] European association for the study of the liver, "EASL clinical practice guidelines on the management of ascites, spontaneous bacterial peritonitis, and hepatorenal syndrome in cirrhosis," Journal of Hepatology, vol. 2010, no. 53, pp. 397-417, 2010.

[7] C. Mortensen, J. S. Jensen, L. Hobolth et al., "Association of markers of bacterial translocation with immune activation in decompensated cirrhosis," European Journal of Gastroenterology \& Hepatology, vol. 26, no. 12, pp. 1360-1366, 2014.

[8] G. Dam, S. Keiding, O. L. Munk et al., "Hepatic encephalopathy is associated with decreased cerebral oxygen metabolism and blood flow, not increased ammonia uptake," Hepatology, vol. 57, no. 1, pp. 258-265, 2013.

[9] H. Vilstrup, P. Amodio, J. Bajaj et al., "Hepatic encephalopathy in chronic liver disease: 2014 Practice Guideline by the American Association for the Study of Liver Diseases and the European Association for the Study of the Liver," Hepatology, vol. 60, no. 2, pp. 715-735, 2014.

[10] E. Kalaitzakis and E. Bjornsson, "Hepatic encephalopathy in patients with liver cirrhosis: is there a role of malnutrition?," World Journal of Gastroenterology, vol. 14, no. 21, pp. 34383439, 2008.

[11] P. Ferenci, A. Lockwood, K. Mullen, R. Tarter, K. Weissenborn, and A. T. Blei, "Hepatic encephalopathy-definition, nomenclature, diagnosis, and quantification: final report of the working party at the 11th World Congresses of Gastroenterology, Vienna, 1998," Hepatology, vol. 35, no. 3, pp. 716-721, 2002.

[12] J. Córdoba, "New assessment of hepatic encephalopathy," Journal of Hepatology, vol. 54, no. 5, pp. 1030-1040, 2011.

[13] R. Rai, V. A. Saraswat, and R. K. Dhiman, "Gut microbiota: its role in hepatic encephalopathy," Journal of Clinical and Experimental Hepatology, vol. 5, Suppl 1, pp. S29-S36, 2015.

[14] Y. Muto, S. Sato, A. Watanabe et al., "Effects of oral branchedchain amino acid granules on event-free survival in patients with liver cirrhosis," Clinical Gastroenterology and Hepatology, vol. 3, no. 7, pp. 705-713, 2005.

[15] M. Guevara, M. E. Baccaro, J. Ríos et al., "Risk factors for hepatic encephalopathy in patients with cirrhosis and refractory ascites: relevance of serum sodium concentration," Liver International, vol. 30, no. 8, pp. 1137-1142, 2010.

[16] C. Bémeur and R. F. Butterworth, "Nutrition in the management of cirrhosis and its neurological complications," Journal of Clinical and Experimental Hepatology, vol. 4, no. 2, pp. 141150, 2014.

[17] L. W. Teperman, "Impact of pretransplant hepatic encephalopathy on liver posttransplantation outcomes," International Journal of Hepatology, vol. 2013, Article ID 952828, 9 pages, 2013.

[18] J. S. Bajaj, J. B. Wade, D. P. Gibson et al., "The multidimensional burden of cirrhosis and hepatic encephalopathy on patients and caregivers," The American Journal of Gastroenterology, vol. 106, no. 9, pp. 1646-1653, 2011.
[19] A. Sanyal, Z. M. Younossi, N. M. Bass et al., "Randomised clinical trial: rifaximin improves health-related quality of life in cirrhotic patients with hepatic encephalopathy - a doubleblind placebo-controlled study," Alimentary Pharmacology \& Therapeutics, vol. 34, no. 8, pp. 853-861, 2011.

[20] J. P. Sturgeon and D. L. Shawcross, "Recent insights into the pathogenesis of hepatic encephalopathy and treatments," Expert Review of Gastroenterology \& Hepatology, vol. 8, pp. 83-100, 2014.

[21] J. S. Bajaj, C. M. Schubert, D. M. Heuman et al., "Persistence of cognitive impairment after resolution of overt hepatic encephalopathy," Gastroenterology, vol. 138, no. 7, pp. 2332-2340, 2010.

[22] C. J. Rosen, J. S. Adams, D. D. Bikle et al., "The nonskeletal effects of vitamin D: an endocrine society scientific statement," Endocrine Reviews, vol. 33, no. 3, pp. 456-492, 2012.

[23] M. S. Calvo, S. J. Whiting, and C. N. Barton, "Vitamin D intake: a global perspective of current status," The Journal of Nutrition, vol. 135, no. 2, pp. 310-316, 2005.

[24] J. Merke, P. Milde, S. Lewicka et al., "Identification and regulation of 1,25-dihydroxyvitamin D3 receptor activity and biosynthesis of 1,25-dihydroxyvitamin D3. Studies in cultured bovine aortic endothelial cells and human dermal capillaries," The Journal of Clinical Investigation, vol. 83, no. 6, pp. 19031915, 1989.

[25] M. Gascon-Barré, C. Demers, A. Mirshahi, S. Néron, S. Zalzal, and A. Nanci, "The normal liver harbors the vitamin D nuclear receptor in nonparenchymal and biliary epithelial cells," Hepatology, vol. 37, no. 5, pp. 1034-1042, 2003.

[26] H. A. Bischoff-Ferrari, M. Borchers, F. Gudat, U. Dürmüller, H. B. Stähelin, and W. Dick, "Vitamin D receptor expression in human muscle tissue decreases with age," Journal of Bone and Mineral Research, vol. 19, no. 2, pp. 265-269, 2004.

[27] D. W. Eyles, S. Smith, R. Kinobe, M. Hewison, and J. J. McGrath, "Distribution of the vitamin D receptor and 1 alpha-hydroxylase in human brain," Journal of Chemical Neuroanatomy, vol. 29, no. 1, pp. 21-30, 2005.

[28] Y. Wang, J. Zhu, and H. F. DeLuca, "Where is the vitamin D receptor?," Archives of Biochemistry and Biophysics, vol. 523, no. 1, pp. 123-133, 2012.

[29] S. Christakos, D. V. Ajibade, P. Dhawan, A. J. Fechner, and L. J. Mady, "Vitamin D: metabolism," Endocrinology and Metabolism Clinics of North America, vol. 39, no. 2, pp. 243-253, 2010.

[30] T. L. Clemens, J. S. Adams, J. M. Nolan, and M. F. Holick, "Measurement of circulating vitamin D in man," Clinica Chimica Acta, vol. 121, no. 3, pp. 301-308, 1982.

[31] E. Borella, G. Nesher, E. Israeli, and Y. Shoenfeld, "Vitamin D: a new anti-infective agent?," Annals of the New York Academy of Sciences, vol. 1317, no. 1, pp. 76-83, 2014.

[32] J. S. Buell and B. Dawson-Hughes, "Vitamin D and neurocognitive dysfunction: preventing "D"ecline?," Molecular Aspects of Medicine, vol. 29, no. 6, pp. 415-422, 2008.

[33] J. C. McCann and B. N. Ames, "Is there convincing biological or behavioral evidence linking vitamin D deficiency to brain dysfunction?," The FASEB Journal, vol. 22, pp. 982-1001, 2008.

[34] C. H. Wilkins, Y. I. Sheline, C. M. Roe, S. J. Birge, and J. C. Morris, "Vitamin D deficiency is associated with low mood and worse cognitive performance in older adults," The American Journal of Geriatric Psychiatry, vol. 14, no. 12, pp. 10321040, 2006. 
[35] J. H. Moon, S. Lim, J. W. Han et al., "Serum 25hydroxyvitamin D level and the risk of mild cognitive impairment and dementia: the Korean Longitudinal Study on Health and Aging (KLoSHA)," Clinical Endocrinology, vol. 83, no. 1, pp. 36-42, 2015.

[36] D. W. Eyles, T. H. Burne, and J. J. McGrath, "Vitamin D, effects on brain development, adult brain function and the links between low levels of vitamin D and neuropsychiatric disease," Frontiers in Neuroendocrinology, vol. 34, no. 1, pp. 47-64, 2013.

[37] C. Annweiler, G. Allali, P. Allain et al., "Vitamin D and cognitive performance in adults: a systematic review," European Journal of Neurology, vol. 16, no. 10, pp. 1083-1089, 2009.

[38] L. R. Harms, T. H. Burne, D. W. Eyles, and J. J. McGrath, "Vitamin D and the brain," Best Practice \& Research. Clinical Endocrinology \& Metabolism, vol. 25, no. 4, pp. 657-669, 2011.

[39] J. Arteh, S. Narra, and S. Nair, "Prevalence of vitamin D deficiency in chronic liver disease," Digestive Diseases and Sciences, vol. 55, no. 9, pp. 2624-2628, 2010.

[40] M. Malham, S. P. Jørgensen, P. Ott et al., "Vitamin D deficiency in cirrhosis relates to liver dysfunction rather than aetiology," World Journal of Gastroenterology, vol. 17, no. 7, pp. 922-925, 2011.

[41] C. S. Stokes, M. Krawczyk, C. Reichel, F. Lammert, and F. Grünhage, "Vitamin D deficiency is associated with mortality in patients with advanced liver cirrhosis," European Journal of Clinical Investigation, vol. 44, no. 2, pp. 176-183, 2014.

[42] K. D. Mullen, "Review of the final report of the 1998 Working Party on definition, nomenclature and diagnosis of hepatic encephalopathy," Alimentary Pharmacology \& Therapeutics, vol. 25, Suppl 1, pp. 11-16, 2007.

[43] P. S. Kamath, R. H. Wiesner, M. Malinchoc et al., "A model to predict survival in patients with end-stage liver disease," Нераtology, vol. 33, no. 2, pp. 464-470, 2001.

[44] C. Infante-Rivard, S. Esnaola, and J. P. Villeneuve, "Clinical and statistical validity of conventional prognostic factors in predicting short-term survival among cirrhotics," Hepatology, vol. 7, no. 4, pp. 660-664, 1987.

[45] Working Group of the Australian and New Zealand Bone and Mineral Society, Endocrine Society of Australia, and Osteoporosis Australia, "Vitamin D and adult bone health in Australia and New Zealand: a position statement," The Medical Journal of Australia, vol. 182, no. 6, pp. 281-285, 2005.

[46] G. D. Carter, "Accuracy of 25-hydroxyvitamin D assays: confronting the issues," Current Drug Targets, vol. 12, no. 1, pp. 19-28, 2011.

[47] T. J. Littlejohns, W. E. Henley, I. A. Lang et al., "Vitamin D and the risk of dementia and Alzheimer disease," Neurology, vol. 83, no. 10, pp. 920-928, 2014.

[48] C. Trautwein, M. Possienke, H. J. Schlitt et al., "Bone density and metabolism in patients with viral hepatitis and cholestatic liver diseases before and after liver transplantation," The American Journal of Gastroenterology, vol. 95, no. 9, pp. 2343-2351, 2000.

[49] H. M. Pappa, E. Bern, D. Kamin, and R. J. Grand, "Vitamin D status in gastrointestinal and liver disease," Current Opinion in Gastroenterology, vol. 24, no. 2, pp. 176-183, 2008.

[50] L. Fisher and A. Fisher, "Vitamin D and parathyroid hormone in outpatients with noncholestatic chronic liver disease," Clinical Gastroenterology and Hepatology, vol. 5, no. 4, pp. 513520, 2007.
[51] C. Konstantakis, P. Tselekouni, M. Kalafateli, and C. Triantos, "Vitamin D deficiency in patients with liver cirrhosis," Annals of Gastroenterology, vol. 29, no. 3, pp. 297-306, 2016.

[52] N. Fernández Fernández, P. Linares Torres, D. Joáo Matias, F. Jorquera Plaza, and J. L. Olcoz Goñi, "Vitamin D deficiency in chronic liver disease, clinical-epidemiological analysis and report after vitamin d supplementation," Gastroenterología y Hepatología, vol. 39, no. 5, pp. 305-310, 2016.

[53] X. Y. Zhao, J. Li, J. H. Wang et al., "Vitamin D serum level is associated with Child-Pugh score and metabolic enzyme imbalances, but not viral load in chronic hepatitis B patients," Medicine, vol. 95, no. 27, article e3926, 2016.

[54] C. Putz-Bankuti, S. Pilz, T. Stojakovic et al., "Association of 25hydroxyvitamin D levels with liver dysfunction and mortality in chronic liver disease," Liver International, vol. 32, no. 5, pp. 845-851, 2012.

[55] H. Vidot, A. Potter, R. Cheng, M. Allman-Farinelli, and N. Shackel, "Serum 25-hydroxyvitamin D deficiency and hepatic encephalopathy in chronic liver disease," World Journal of Hepatology, vol. 9, no. 10, pp. 510-518, 2017.

[56] P. Iruzubieta, "Vitamin D deficiency in chronic liver disease," World Journal of Hepatology, vol. 6, no. 12, pp. 901-915, 2014.

[57] E. Trépo, R. Ouziel, P. Pradat et al., "Marked 25hydroxyvitamin D deficiency is associated with poor prognosis in patients with alcoholic liver disease," Journal of Hepatology, vol. 59, no. 2, pp. 344-350, 2013.

[58] F. Finkelmeier, B. Kronenberger, V. Köberle et al., "Severe 25hydroxyvitamin D deficiency identifies a poor prognosis in patients with hepatocellular carcinoma - a prospective cohort study," Alimentary Pharmacology \& Therapeutics, vol. 39, no. 10, pp. 1204-1212, 2014.

[59] R. Paternostro, D. Wagner, T. Reiberger et al., "Low 25-OHvitamin $\mathrm{D}$ levels reflect hepatic dysfunction and are associated with mortality in patients with liver cirrhosis," Wiener Klinische Wochenschrift, vol. 129, no. 1-2, pp. 8-15, 2017.

[60] Z. Bai, X. Guo, F. Tacke, Y. Li, H. Li, and X. Qi, "Association of serum albumin level with incidence and mortality of overt hepatic encephalopathy in cirrhosis during hospitalization," Therapeutic Advances in Gastroenterology, vol. 12, pp. 1-14, 2019. 